PROCEEDINGS OF THE

AMERICAN MATHEMATICAL SOCIETY

Volume 136, Number 8, August 2008, Pages 2815-2822

S 0002-9939(08)09440-9

Article electronically published on April 14, 2008

\title{
LOCAL AUTOMORPHISMS OF THE HILBERT BALL
}

\author{
BERNHARD LAMEL \\ (Communicated by Mei-Chi Shaw)
}

\begin{abstract}
Every holomorphic mapping which takes a piece of the boundary of the unit ball in complex Hilbert space into the boundary of the unit ball and whose differential at some point of this boundary is onto is the restriction of an automorphism of the ball. We also show that it is enough to assume that the mapping is only Gâteaux-holomorphic.
\end{abstract}

\section{INTRODUCTION AND STATEMENT OF RESULTS}

Let $\mathbb{B}_{N} \subset \mathbb{C}^{N}$ be the unit ball in $\mathbb{C}^{N}$; that is,

$$
\mathbb{B}_{N}=\left\{Z \in \mathbb{C}^{N}:\|Z\|<1\right\} .
$$

It is well known that if $H:\left(U, p_{0}\right) \rightarrow \mathbb{C}^{N}$ is a holmorphic mapping defined in a neighbourhood $U \subset \mathbb{C}^{N}$ of some $p_{0} \in \partial \mathbb{B}_{N}$ which maps $U \cap \partial \mathbb{B}_{N}$ into $\partial \mathbb{B}_{N}$, then $H$ is either a constant mapping or it extends to an automorphism of $\mathbb{B}_{N}$. This theorem is due to Alexander [1].

In a Hilbert space of infinite dimension, Alexander's Theorem is not valid (see Example 1: the author is grateful to Laszlo Lempert for pointing out this fact). However, if we assume in addition that the differential of the mapping $H$ (see Theorem 10 is onto at some point $p_{0}$ on the boundeary of the ball, then the same extension property as in finite dimensional Hilbert space holds. That is, a mapping $H$ defined near a point $p_{0}$ in the boundary of the unit ball $\mathcal{B} \subset \mathcal{H}$ of some complex Hilbert space $\mathcal{H}$ whose differential at $p$ is onto extends to an automorphism of $\mathcal{B}$. In order to formulate our result, we recall that a mapping $H: U \rightarrow \mathcal{H}$ defined on some open subset $U \subset \mathcal{H}$ is Gâteaux-holomorphic if for all $\tilde{Z} \in \mathcal{H}$, the function $Z \mapsto\langle H(Z), \tilde{Z}\rangle$ is holomorphic on all sets of the form $E \cap U$, where $E$ is some finite dimensional, affine subspace of $\mathcal{H}$. A Gâteaux-holomorphic map $H$ is holomorphic if it is also continuous (for these definitions, see e.g. the book of Dineen [4]). We can now state our main result.

Theorem 1. Let $\mathcal{H}$ be a complex Hilbert space, $P \in \mathcal{H}$ with $\|P\|=1$, and $H: U \rightarrow \mathcal{H}$ be a Gâteaux-holomorphic map defined on a neigbourhood $U$ of $P$ with the property that $\|H(Z)\|=1$ for all $Z \in U$ with $\|Z\|=1$, and such that the range of $H^{\prime}(P)$ is $\mathcal{H}$. Then $H$ is holomorphic, and furthermore, $H$ extends to an automorphism of $\mathcal{B}$.

Received by the editors November 21, 2006.

2000 Mathematics Subject Classification. Primary 32H12, 46G20, 46T25, 58C10.

The author was supported by the Austrian Science Fund FWF, Projects P17111 and P19667.

(C) 2008 American Mathematical Society Reverts to public domain 28 years from publication 
Note that we do not assume any continuity of the map here; it is indeed part of the conclusion that the mapping $H$ is continuous. Also note the assumption of Gâteaux-holomorphicity implies that $H$ has a weak (not a priori continuous) derivative, which is defined on all of $\mathcal{H}$; it is this derivative that we denote by $H^{\prime}(P)$ in the statement of Theorem 1. It follows that $H^{\prime}(P) v$ is given by the strong limit of the difference quotients,

$$
H^{\prime}(P) v=\lim _{\mathbb{C} \ni t \rightarrow 0} \frac{H(P+t v)-H(P)}{t} .
$$

We do not assume a priori that $H^{\prime}(P)$ is bounded; similar remarks hold for higher derivatives. We note that the automorphisms of a ball in Hilbert space are well known (this goes back to a paper of Renaud [5) and that any such automorphism is a linear fractional map (just as in the finite dimensional case) which extends holomorphically across the boundary of the ball.

The proof of Theorem 1 is an application of the technique of Segre varieties on the Hilbert space in question. The special structure of the boundary of the ball allows us to do the necessary polarization very explicitly and also allows us to exploit the Riesz Representation Theorem (that is, the self-duality of the Hilbert space) to explicitly calculate expressions for $H$. We will do this computation in a set of different coordinates which are used for computations of this type in finite dimensional complex space, the so-called "normal coordinates". In the case of the unit ball, its boundary is put into normal coordinates by the well-known Cayley transform. Our calculations are very explicit, and also yield a good understanding of the structure of the automorphism group in question.

In our proof, we will pass back and forth between applying the Segre technique along finite dimensional affine subspaces and using the results of these calculations to get global expressions for the map. It is this passing back and forth that allows us to establish holomorphicity of the map without assuming continuity. The technique of the Segre varieties has been used extensively in the study of holomorphic mappings in the finite dimensional case; for a detailed discussion of their use in that case, we refer the reader to e.g. the survey article of Baouendi, Ebenfelt and Rothschild [3].

Algorithmically, our calculations follow along the path where Baouendi, Ebenfelt and Rothschild 2] established that automorphisms of real-analytic hypersurfaces in $\mathbb{C}^{N}$ are parametrized by their jets of some finite order. Our calculations here also yield an explicit parametrization for the group of local automorphisms of the boundary of the unit ball in $\mathcal{H}$; see (2) below.

As noted above, the change to normal coordinates is given by means of the Cayley transform, which is recalled in Section 2. In these coordinates, given a point $P \in \partial \mathcal{B}, \mathcal{B}$ corresponds to

$$
\mathbb{H}_{+}=\left\{(z, w): \operatorname{Im} w>\|z\|^{2}\right\} \subset \mathbb{C} \times P^{\perp}
$$

and $P$ corresponds to $(0,0)$. The boundary $\partial \mathcal{B}$ corresponds to

$$
\mathbb{H}=\left\{(z, w): \operatorname{Im} w=\|z\|^{2}\right\} .
$$

Theorem 1 is a consequence of the following, more detailed theorem.

Theorem 2. Let $F$ be a Hilbert space, $H: U \rightarrow F \times \mathbb{C}$ a map defined in a neighbourhood $U$ of $(0,0)$ in $F \times \mathbb{C}$ which is Gâteaux-holomorphic on $U$ (that is, for every 
affine subspace $E$ of $F \times \mathbb{C}$ and for every continuous linear form $\phi$ on $F \times \mathbb{C}$, the map $\phi(H): E \cap U \rightarrow \mathbb{C}$ is holomorphic), satisfies $H(0)=0$, and $H(U \cap \mathbb{H}) \subset \mathbb{H}$. Assume in addition that the range of $H^{\prime}(0)$ is $\mathcal{H}$. Then $H$ is holomorphic, and furthermore, if we denote the coordinate in $F$ by $z$ and the coordinate in $\mathbb{C}$ by $w$, then

$$
H(Z)=\left(s U \frac{z+a w}{1-2 i\langle z, a\rangle+\left(R-i\|a\|^{2}\right) w}, s^{2} \frac{w}{1-2 i\langle z, a\rangle+\left(R-i\|a\|^{2}\right) w}\right),
$$

where $U$ is a unitary map, $s \in \mathbb{R}_{+}, a \in \mathbb{C}, R \in \mathbb{R}$, and $U, s, a, R$ are given by

$$
\begin{aligned}
U & =\frac{f_{z}(0)}{\sqrt{g_{w}(0)}}, & s & =\sqrt{g_{w}(0)}, \\
a & =-f_{z}(0)^{-1} f_{w}(0), & R & =\frac{2 g_{w^{2}}(0)+i\left\|f_{w}(0)\right\|^{2}}{g_{w}(0)} .
\end{aligned}
$$

It is natural to ask whether or not the assumption on the range of $H^{\prime}(P)$ is necessary. The simplest example of a map violating this condition, which is not an automorphism of $\mathcal{B}$, was pointed out to the author by Lempert: Just consider an isometric map of $\mathcal{H}$ onto a proper subspace of itself (e.g. the shift map on $\ell^{2}$ ).

There are numerous other interesting examples of maps whose derivative is not onto at any point on the boundary; let us discuss two of them here.

Example 1. Let $\mathcal{H}=\ell^{2}$, and write $Z=\left(z_{1}, z_{2}, \cdots\right)$ for the variable in $\ell^{2}$. Consider the set

$$
\Gamma=\bigcup_{k \in \mathbb{N}} \mathbb{N}^{k}
$$

This is a countable set; choose a bijection $\phi: \Gamma \rightarrow \mathbb{N}$. Let $\lambda_{j}$ be a sequence with

$$
\sum_{j}\left|\lambda_{j}\right|^{2}=1
$$

For $\alpha=\left(\alpha_{1}, \cdots, \alpha_{k}\right) \in \Gamma$, write $z_{\alpha}=z_{\alpha_{1}} \cdots z_{\alpha_{n}}$ and $|\alpha|=k$. We define $H(Z)=$ $W=\left(w_{1}, w_{2}, \cdots\right)$ by setting $w_{\phi(\alpha)}=\lambda_{|\alpha|} z_{\alpha}$. Then $H$ maps $r \mathcal{B}$ into $R(r) \mathcal{B}$ for $0 \leq r<r_{0}$ for some $r_{0}>1$, where

$$
R(r)=\sum_{j}|\lambda|^{2} r^{j}
$$

It is easy to show that $H$ is a holomorphic map; it is clearly not an automorphism.

Example 2. Our second example generalizes the Whitney map. Using the notation introduced above, for a given $p \in \mathbb{N} \cup\{\infty\}$, we now consider as an index set

$$
\begin{gathered}
\Gamma_{p}=\bigcup_{0 \leq q<p}\{q\} \times\{j \in \mathbb{N}: j>1\} \cup\{p\}, \quad p<\infty, \\
\Gamma_{\infty}=\bigcup_{0 \leq q}\{q\} \times\{j \in \mathbb{N}: j>1\} .
\end{gathered}
$$

Again, we choose a bijection $\phi: \Gamma_{p} \rightarrow \mathbb{N}$ and we define $W=H(Z)$ by

$$
w_{\phi(q, k)}=z_{1}^{q} z_{k}, \quad w_{\phi(p)}=z_{1}^{p} \text { in case } p<\infty .
$$


Then we have that

$$
\begin{gathered}
\|H(Z)\|^{2}=\frac{1-\left|z_{1}\right|^{2 p}}{1-\left|z_{1}\right|^{2}}\left(\|Z\|^{2}-\left|z_{1}\right|^{2}\right)+\left|z_{1}\right|^{2 p}, \quad p \neq \infty, \\
\|H(Z)\|^{2}=\frac{1}{1-\left|z_{1}\right|^{2}}\left(\|Z\|^{2}-\left|z_{1}\right|^{2}\right), \quad p=\infty .
\end{gathered}
$$

It is easy to check that if $p \neq \infty, H$ extends holomorphically to a neighbourhood of $\overline{\mathcal{B}}$. On the other hand, if $p=\infty, H$ does not extend holomorphically to any neighbourhood of $(1,0, \cdots)$ (indeed, it does not even extend continuously up to that point!).

\section{Changing coordinates and some observations}

We now assume that we have the following situation: $H$ is a map defined in a neighbourhood $U$ of $P$, satisfying the assumptions of Theorem 1 After composing with a rotation in the plane spanned by $P$ and $H(P)$, we can assume that $H(P)=$ $P$. We decompose $\mathcal{H}=F \oplus \mathbb{C} P$, where $F=P^{\perp}$. In this decomposition, we write $Z=(\zeta, \eta)$ with $\zeta \in F$ and $\eta \in \mathbb{C}$. Our goal in this section is to show that Theorem 2 implies Theorem 1 and to assemble the necessary prerequisites for the proof of Theorem 2 which will be given in the following section.

2.1. The Cayley transform. The Cayley transform (resp. its inverse) is defined by

$$
(z, w)=\left(\frac{\zeta}{1+\eta}, i \frac{1-\eta}{1+\eta}\right), \quad(\zeta, \eta)=\left(\frac{2 i z}{i+w}, \frac{i-w}{i+w}\right)
$$

and is a local biholomorphism from a neigbourhood of $P$ to $F \oplus \mathbb{C}$, taking $P$ to $(0,0)$ and the boundary of the unit ball (with the point $-P$ omitted) to the hypersurface $\mathbb{H}$ in $F \oplus \mathbb{C}$ defined by the equation

$$
\operatorname{Im} w=\|z\|^{2}, \quad(z, w) \in F \oplus \mathbb{C} .
$$

It also takes the interior of the unit ball to the half-space $\mathbb{H}_{+}=\left\{\operatorname{Im} w \geq\|z\|^{2}\right\}$.

Employing the Cayley transform, we see that Theorem 2 implies Theorem[1. So from now on, we assume that $H$ is defined in a neighbourhood of $(0,0) \in F \oplus \mathbb{C}$, takes $(0,0)$ to $(0,0)$, and if we write $H(z, w)=(f(z, w), g(z, w))$ with $f(z, w) \in F$ and $g(z, w) \in \mathbb{C}$, then we have

$$
\operatorname{Im} g(z, w)=\|f(z, w)\|^{2}, \text { if } \operatorname{Im} w=\|z\|^{2} .
$$

2.2. Complexification. We will now complexify the last equation (in a bit of a nonstandard manner, owing to the fact that the natural isomorphism $\mathcal{H} \rightarrow \mathcal{H}^{*}$ induced by the inner product is conjugate linear). We claim that we have

$$
g(z, w)-\overline{g(\chi, \tau)}=2 i\langle f(z, w), f(\chi, \tau)\rangle, \text { if } w-\bar{\tau}=2 i\langle z, \chi\rangle .
$$

We check this claim by restricting ourselves to the finite dimensional subspace spanned by $z$ and $\chi$ : Choose an orthonormal basis $\{u, v\}$ of the span of $z$ and $\chi$. We have

$$
z=r_{z} u+s_{z} v, \quad \chi=r_{\chi} u+s_{\chi} v
$$

and consider the hypersurface $M$ in $\mathbb{C}^{3}$ with coordinates $(r, s, w)$ defined by

$$
\operatorname{Im} w=|r|^{2}+|s|^{2} .
$$


On this hypersurface, consider the function $\rho$ defined by

$$
\rho(r, s, w)=\operatorname{Im} g(r u+s v, w)-\langle f(r u+s v, w), f(z, w)\rangle, \quad z=r u+s v .
$$

A fact which we are going to use often in the following is that the Banach-Steinhaus Theorem guarantees that a mapping which is Gâteaux-holomorphic is automatically uniformly bounded along compact subsets of finitely dimensional affine subspaces of $\mathcal{H}$. This in turn implies that the function $\rho$ is real-analytic on a neighbourhood of 0 in $\mathbb{C}^{3}$; by assumption it vanishes on $M$, so the usual complexification shows that

$g(r u+s v, w)-\overline{g(p u+q v, \tau)}-2 i\langle f(r u+s v, w), f(p u+q v, \tau)\rangle$ if $w-\bar{\tau}=2 i(r \bar{p}+s \bar{q})$.

2.3. CR and transversal vector fields. Our next step is to introduce two vector fields which we are going to use to differentiate (3). We choose an $u \in F$ and define

$$
\mathcal{L}_{u}=\frac{\partial}{\partial \bar{u}}-2 i\langle z, u\rangle \frac{\partial}{\partial \bar{\tau}} .
$$

The application of $\mathcal{L}_{u}$ to a function $\phi$ is defined in the following natural way: Suppose that $\phi(z, w, \chi, \tau)$ is valued in some Hilbert space $E$, defined in a neighbourhood of 0 and that we are given $z_{0}, w_{0}, \chi_{0}$ and $\tau_{0}$. We choose an orthonormal basis $\{u, g, h\}$ of the space spanned by $z_{0}, \chi_{0}$ and $u$. Setting $z=r u+s g+t h$ and $\chi=a u+b g+c h$, we define $\mathcal{L}_{u} \phi\left(z_{0}, w_{0}, \chi_{0}, \tau_{0}\right)$ to be the application of the vector field

$$
\frac{\partial}{\partial \bar{a}}-2 i r \frac{\partial}{\partial \bar{\tau}}
$$

to $\phi$ at the appropriate point.

We also define the vector field

$$
\mathcal{X}=\frac{\partial}{\partial w}+\frac{\partial}{\partial \bar{\tau}} .
$$

Remark 1. Note that the Banach-Steinhaus Theorem implies that given a map $h$ which is a Gâteaux-holomorphic map from a Hilbert space $\mathcal{H}_{1}$ into some other Hilbert space $\mathcal{H}_{2}, h$ possesses all weak directional derivatives. By this we mean the following: For each $u \in \mathcal{H}_{1}$ and each $u_{0}$ in the domain of $h$, there exists a vector $v \in \mathcal{H}_{2}$ such that for all $P \in \mathcal{H}_{2}$,

$$
\lim _{t \rightarrow 0} \frac{\left\langle h\left(u_{0}+t u\right), P\right\rangle-\left\langle h\left(u_{0}\right), P\right\rangle}{t}=\langle v, P\rangle .
$$

It follows by well-known arguments that this limit is actually strong; that is,

$$
v=\lim _{t \rightarrow 0} \frac{h\left(u_{0}+t u\right)-h\left(u_{0}\right)}{t} .
$$

In this situation, we will write $v=h^{\prime}\left(u_{0}\right)(u)$. Note that we do not assume that $h$ is differentiable when we use this notation; also, $h^{\prime}\left(u_{0}\right)$ is not necessarily bounded. In the case that we are dealing with, we will write

$$
f_{z}\left(z_{0}, w_{0}\right) u=f^{\prime}\left(z_{0}, w_{0}\right)(u, 0),
$$

and so on; we will identify e.g. $f_{w}\left(z_{0}, w_{0}\right)$ with the vector $f_{w}\left(z_{0}, w_{0}\right)(0,1)$ and the derivatives $g_{w}\left(z_{0}, w_{0}\right)$ with number $g_{w}\left(z_{0}, w_{0}\right)(0,1)$. Let us emphasize again that writing $f_{z}$ does not mean we assume that $f$ is differentiable (that's part of what we want to show). 


\section{Proof of Theorem 2}

We now start by applying $\mathcal{L}_{u}$ to (3). We obtain

$$
-\overline{g_{z}(\chi, \tau) u+2 i\langle u, z\rangle g_{w}(\chi, \tau)}=2 i\left\langle f(z, w), f_{z}(\chi, \tau) u+2 i\langle u, z\rangle f_{\tau}(\chi, \tau)\right\rangle,
$$

which is valid for $w-\bar{\tau}=2 i\langle z, \chi\rangle$ and every $u \in F$. Setting $\chi=0, \tau=w=0$ and noting that (3) implies $g(z, 0)=0$, we get that

$$
\overline{g_{w}(0)}\langle z, u\rangle=\left\langle f(z, 0), f_{z}(0) u+2 i\langle u, z\rangle f_{w}(0)\right\rangle,
$$

which holds for every $z \in F$ close enough by 0 and every $u \in F$. In particular, we have

$$
\overline{g_{w}(0)}\langle u, v\rangle=\left\langle f_{z}(0) u, f_{z}(0) v\right\rangle .
$$

So, if the range of $H^{\prime}(P)$ is $\mathcal{H}$, this implies that $f_{z}(0)$ is an isomorphism. Furthermore, in that case, $g_{w}(0)=r^{2}$ is a positive real number, and the map $r^{-1} f_{z}(0)$ is unitary. Consider the automorphisms $\omega_{U, s}$ for unitary operators $U$ of $F$ and $s \in \mathbb{R}$ defined by

$$
\omega_{U, s}(z, w)=\left(s U z, s^{2} w\right) .
$$

Composing with the automorphism $(z, w) \mapsto \omega_{r f_{z}(0)^{-1}, r^{-1}}(z, w)=\left(f_{z}(0)^{-1} z, r^{-2} w\right)$, we can assume that $f_{z}(0)=I$ and $g_{w}(0)=1$.

Returning to (6), we note that we can now explicitly compute $f(z, 0)$. Indeed, with the simplifications made above, we have

$$
\langle z, u\rangle=\left\langle f(z, 0), u+2 i\langle u, z\rangle f_{w}(0)\right\rangle
$$

for $z \in F$ close by 0 , and all $u \in F$. Writing $T u=u+2 i\langle u, z\rangle f_{w}(0)$, we conclude that $f(z, 0)=T^{*-1} z$, and computing $T^{*-1} v$ we obtain

$$
f(z, 0)=\frac{z}{1+\left\langle z, 2 i f_{w}(0)\right\rangle} .
$$

The next simplification we can make is that we can assume $f_{w}(0)=0$. Indeed, for $a \in F$, the automorphism $\phi_{a}$ defined by

$$
\phi_{a}(z, w)=\left(\frac{z+w a}{1-2 i\langle z, a\rangle-i w\|a\|^{2}}, \frac{w}{1-2 i\langle z, a\rangle-i w\|a\|^{2}}\right)
$$

takes $\mathbb{H}$ into itself. Composing with $\phi_{a}$ for $a=-f_{w}(0)$ we see that we can actually assume $f_{w}(0)=0$.

In the next step, we first apply $\mathcal{X}$ to (3) to obtain

$$
g_{w}(z, w)-\overline{g_{w}(\chi, \tau)}=2 i\left(\left\langle f_{w}(z, w), f(\chi, \tau)\right\rangle+\left\langle f(z, w), f_{w}(\chi, \tau)\right\rangle\right) .
$$

Evaluating again at $\chi=0, \tau=w=0$, we get $g_{w}(z, 0)=\overline{g_{w}(0)}$. Now we apply $\mathcal{L}_{u}$ to (9) and evaluate at $\chi=0, w=\tau=0$. The result is

$$
\overline{g_{w^{2}}(0)}\langle z, u\rangle=\left\langle f_{w}(z, 0), u\right\rangle+\left\langle z, f_{z w}(0) u+2 i\langle u, z\rangle f_{w^{2}}(0)\right\rangle,
$$

from which we see that $f_{z w}(0)^{*} z$ is defined for all $z \in F$ (since the equation exhibits that $u \mapsto\left\langle z, f_{z, w}(0) u\right\rangle$ is continuous for $z$ close by 0$)$. We have

$$
f_{w}(z, 0)=\overline{g_{w^{2}}(0)} z-f_{z w}^{*}(0) z-\left\langle z, 2 i f_{w^{2}}(0)\right\rangle z .
$$


Now turn back to (5) and evaluate this equation at $\tau=0, w=2 i\langle z, \chi\rangle$. Applying our simplifications, it then reads

$$
\begin{aligned}
\langle z, u\rangle & =\overline{g_{w}(0)}\langle z, u\rangle \\
& =\overline{g_{w}(\chi, 0)}\langle z, u\rangle \\
& =\left\langle f(z, 2 i\langle z, \chi\rangle), f_{z}(\chi, 0) u+2 i\langle u, z\rangle f_{w}(\chi, 0)\right\rangle \\
& =\left\langle f(z, 2 i\langle z, \chi\rangle), u+2 i\langle u, z\rangle f_{w}(\chi, 0)\right\rangle .
\end{aligned}
$$

We note that $T u=u+2 i\langle u, z\rangle f_{w}(\chi, 0)$ is a continuous, invertible linear map for $z$ and $\chi$ close by 0 , and compute

$$
T^{*-1} v=v-\frac{\left\langle v, 2 i f_{w}(\chi, 0)\right\rangle}{1+\left\langle z, 2 i f_{w}(\chi, 0)\right\rangle} z .
$$

With this observation, (12) implies that

$$
\begin{aligned}
f(z, 2 i\langle z, \chi\rangle) & =T^{*-1} z \\
& =\frac{z}{1+\left\langle z, 2 i f_{w}(\chi, 0)\right\rangle} \\
& =\frac{z}{1-2 i g_{w^{2}}(0)\langle z, \chi\rangle+2 i\left\langle z, f_{z w}(0)^{*} \chi\right\rangle-4\langle z, \chi\rangle\left\langle f_{w^{2}}(0), \chi\right\rangle}
\end{aligned}
$$

and

$$
g(z, 2 i\langle z, \chi\rangle)=\frac{2 i\langle z, \chi\rangle}{1-2 i g_{w^{2}}(0)\langle z, \chi\rangle+2 i\left\langle z, f_{z w}(0)^{*} \chi\right\rangle-4\langle z, \chi\rangle\left\langle f_{w^{2}}(0), \chi\right\rangle} .
$$

We now claim that $f_{w^{2}}(0)=0$. To check this, we have to use Gâteaux-holomorphicity once more. We choose a $\chi_{0} \in F$, and for $z$ with $\left\langle z, \chi_{0}\right\rangle \neq 0$ we set $w=$ $2 i\left\langle z, \lambda \chi_{0}\right\rangle$, getting $\lambda=\frac{i \bar{w}}{2\left\langle\chi_{0}, z\right\rangle}$. We also set $\left\langle z, f_{z w}(0)^{*} \chi_{0}\right\rangle=a\left(\chi_{0}\right)\left\langle z, \chi_{0}\right\rangle$. Hence,

$$
\begin{aligned}
& f(z, w)=\frac{z}{1-\left(g_{w^{2}}(0)-a\left(\chi_{0}\right)\right) w-w^{2} \frac{\left\langle f_{w^{2}}(0), \chi_{0}\right\rangle}{\left\langle z, \chi_{0}\right\rangle}}, \\
& g(z, w)=\frac{w}{1-\left(g_{w^{2}}(0)-a\left(\chi_{0}\right)\right) w-w^{2} \frac{\left\langle f_{w^{2}}(0), \chi_{0}\right\rangle}{\left\langle z, \chi_{0}\right\rangle}} .
\end{aligned}
$$

Since $H$ is Gâteaux-holomorphic, the map

$$
\psi(\lambda, \mu)=g\left(\lambda \chi_{0}, \mu\right)=\frac{\mu}{1-\left(g_{w^{2}}(0)-a\left(\chi_{0}\right)\right) \mu-\frac{\mu^{2}}{\lambda} \frac{\left\langle f_{w^{2}}(0), \chi_{0}\right\rangle}{\left\|\chi_{0}\right\|^{2}}}
$$

is a holomorphic map $\mathbb{C}^{2} \rightarrow \mathbb{C}$ (a priori, the equation only holds on an open subset of $\mathbb{C}^{2}$, of course, but this implies that the functions coincide as rational functions). This of course is only possible if the coefficient of $\frac{\mu^{2}}{\lambda}$ in the denominator does vanish, that is, if $\left\langle f_{w^{2}}(0), \chi_{0}\right\rangle=0$. Since this holds for all $\chi_{0}$ sufficiently close by 0 , we conclude that $f_{w^{2}}(0)=0$. Thus, (13) becomes

$$
\begin{aligned}
& f(z, w)=\frac{z}{1-\left(g_{w^{2}}(0)-a\left(\chi_{0}\right)\right) w}, \\
& g(z, w)=\frac{w}{1-\left(g_{w^{2}}(0)-a\left(\chi_{0}\right)\right) w} .
\end{aligned}
$$

This holds for all $(z, w)$ close by the origin which are of the form $w=2 i\langle z, \chi\rangle$ for some $\chi$ close by the origin. Since this definitely includes an open set in each finite dimensional affine subspace, the principle of analytic continuation and Gâteauxholomorphicity of $H$ implies that (14) holds for all $(z, w)$ close by the origin. At this 
point, we have thus established that $H$ is actually holomorphic in a neighbourhood of the origin. Taking the second derivative with respect to $w$ of the equation for $g$ in (14), we see that $a\left(\chi_{0}\right)=\frac{g_{w^{2}}(0)}{2}$, so that $f_{z w}(0) u=\frac{g_{w^{2}}(0)}{2} u$, and we conclude that

$$
\begin{aligned}
& f(z, w)=\frac{z}{1+R w}, \\
& g(z, w)=\frac{w}{1+R w},
\end{aligned}
$$

where $R=-\frac{g_{w^{2}}(0)}{2}$. Next, let us check that $H$ leaving $\mathbb{H}$ invariant implies that $R \in \mathbb{R}$. We have

$$
\begin{aligned}
\operatorname{Im} \frac{w}{1+R w} & =\frac{\operatorname{Im} w}{|1+R w|^{2}}-\frac{|w|^{2}(\operatorname{Im} R)}{|1+R w|^{2}} \\
& =\frac{\|z\|^{2}}{|1+R w|^{2}},
\end{aligned}
$$

which implies that $\operatorname{Im} R=0$. So, we have $g_{w^{2}}(0) \in \mathbb{R}$. Thus, the denominator of our expressions for $f$ and $g$ do not vanish on the set $\mathbb{H}_{+}=\left\{\operatorname{Im} w \geq\|z\|^{2}\right\}$; thus an $H$ of the form above is holomorphic on all of $\mathbb{H}_{+}$.

We will now recover the general form of a map $H$ by retracing the simplifications made above. Let us write $H_{R}$ for the map given by (15). We have to compose $H_{R}$ with a map of the form $\phi_{a}$ and a map of the form $\omega_{U, s}$. This yields as the general form of an automorphism

$$
\omega_{U, s} \phi_{a} H_{R}=\left(s U \frac{z+a w}{1-2 i\langle z, a\rangle+\left(R-i\|a\|^{2}\right) w}, s^{2} \frac{w}{1-2 i\langle z, a\rangle+\left(R-i\|a\|^{2}\right) w}\right) .
$$

We are only missing the last conclusion of Theorem 2, In order to prove this, just note that the particular choice of the maps made above implies that we can compute $U, s, a$ and $R$ from the derivatives of our original map $H$ in the following way:

$$
\begin{aligned}
U & =\frac{f_{z}(0)}{\sqrt{g_{w}(0)}}, & s & =\sqrt{g_{w}(0)}, \\
a & =-f_{z}(0)^{-1} f_{w}(0), & R & =\frac{2 g_{w^{2}}(0)+i\left\|f_{w}(0)\right\|^{2}}{g_{w}(0)} .
\end{aligned}
$$

\section{REFERENCES}

1. H. Alexander, Holomorphic mappings from the ball and polydisc, Math. Ann. 209 (1974), 249-256. MR0352531 (50:5018)

2. M. S. Baouendi, P. Ebenfelt, and L. P. Rothschild, Parametrization of local biholomorphisms of real analytic hypersurfaces, Asian J. Math. 1 (1997), no. 1, 1-16. MR99b:32022

3. L Local geometric properties of real submanifolds in complex space, Bull. Amer. Math. Soc. (N.S.) 37 (2000), no. 3, 309-336 (electronic). MR2001a:32043

4. S. Dineen, Complex analysis on infinite-dimensional spaces, Springer Monographs in Mathematics, Springer-Verlag London Ltd., London, 1999. MR.1705327(2001a:46043)

5. A. Renaud, Quelques propriétés des applications analytiques d'une boule de dimension infinie dans une autre, Bull. Sci. Math. (2) 97 (1973), 129-159 (1974). MR0338455 (49:3219)

Fakultät für Mathematik, Universität Wien, Nordbergstrasse 15, A-1090 Wien, ÖSTERREICH

E-mail address: bernhard.lamel@univie.ac.at 